\title{
Characteristics of GaAs HEMTs with Flip-Chip Interconnections
}

\author{
Naoko Ono, Fumio Sasaki*, Kazuhiro Arai*, and Yuji Iseki \\ Corporate Research \& Development Center, Toshiba Corporation \\ *Komukai Operations, Toshiba Corporation \\ 1, Komukai Toshiba-cho, Saiwai-ku, Kawasaki 212-8582, Japan \\ E-mail: nao.ono@toshiba.co.jp
}

A GaAs HEMT with flip-chip interconnections has been developed. There are various ground current passes for the HEMT on surface of the GaAs chip in this assembly structure, each pass depending on the transmission line type for the chip. We evaluated the high-frequency characteristics of the HEMT TEGs with flip-chip interconnection for three types of the transmission lines: with a microstrip line (MSL), with a coplanar waveguide (CPW), and with an inverted microstrip line (IMSL). All three types of TEGs had similar values of a maximum available power gain (MAG) at 30 GHz. However, the IMSL-type TEG, which had superior characteristics in high-frequency ranges of more than $30 \mathrm{GHz}$, was chosen as the most suitable type. The IMSL-type TEG had an MAG of $10.02 \mathrm{~dB}$ and a Rollett stability factor $\mathrm{K}$ of 1.20 at $30 \mathrm{GHz}$.

\section{INTRODUCTION}

A flip-chip interconnection structure, in which a semiconductor chip and an assembly substrate are connected using bumps, is expected to become increasingly popular for high-frequency devices such as GaAs high electron mobility transistor (HEMT) [1-5], because the structure has merits such as excellent highfrequency characteristics, small size and low cost. However, the structure with flip-chip interconnections is very different from the conventional structure using bonding wires. In particular, there are various ground current passes for the active device on surface of the GaAs chip in the structure with flip-chip interconnections, each pass depending on the transmission line type for the chip. In addition, active devices on surface of the chip face the surface of assembly substrate directly, and so the devices are influenced strongly by the substrate. Therefore, it is necessary to choose a suitable transmission line for the chip.

K-band systems such as fixed wireless access (FWA) systems have spread throughout the world in recent years, because it is possible to construct wideband access rapidly at a low cost. In particular, point-tomultipoint (PTMP) systems are attracting attention for last/first mile solution since it is possible to install a wideband subscriber loop easily. In order to expand this system, it is necessary to reduce the size and cost of outdoor units (ODUs) [6]. Therefore, the flip-chip interconnection technology for high-frequency active device is very attractive for these applications.

We have developed a GaAs HEMT with flip-chip interconnections. We evaluate in detail the highfrequency characteristics of the HEMT test element groups (TEGs) with flip-chip interconnection using different types of transmission lines for the chip, and choose the most suitable type.

\section{ASSEMBLY STRUCTURE}

\subsection{HEMT ASSEMBLY STRUCTURE}

Figure 1 shows the cross-sectional view of the HEMT assembly structures. The cut plane of Fig. 1 is set along the signal line pattern.

Conventional assembly structure is shown in Fig. 1 (a). The semiconductor chip is mounted on the assembly substrate with face-up structure, and the chip and the substrate are connected using bonding-wires. The amount of extrinsic components for interconnection is large, because of long bonding-wire length. A microstrip line (MSL) and a coplanar waveguide (CPW) are typical transmission lines for the chip.

In the assembly structure with flip-chip interconnections, which is shown in Fig. 1 (b), the GaAs chip was mounted on the surface of the assembly substrate with flip-chip interconnections using bumps. The distance between the electrode for the semiconductor 
chip and the electrode for the assembly substrate is short, and so the amount of extrinsic components for interconnection is small. The flip-chip interconnection utilizes the underfill resin between the chip and the substrate [7]. Because the underfill resin is effective in relaxing the thermal stress between the chip and the substrate and in encapsulating the chip, the structure achieves high reliability.

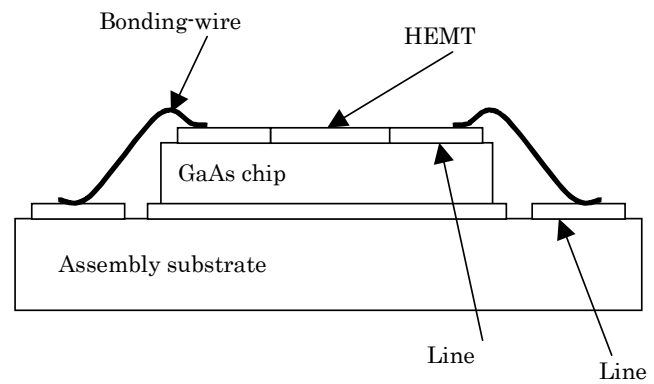

(a) Conventional assembly structure

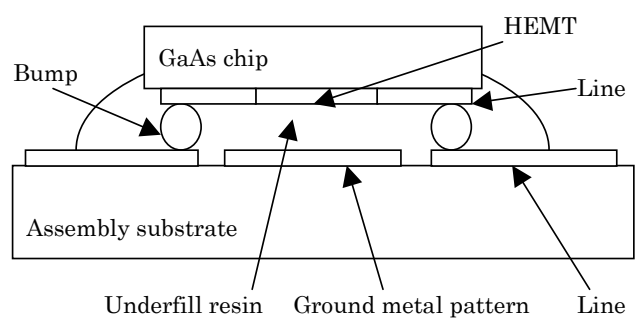

(b) Flip-chip interconnection assembly structure

Figure 1: Cross-sectional view of the HEMT assembly structures

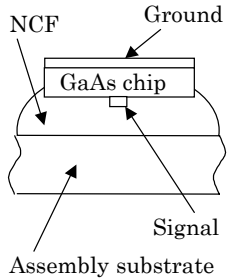

(a) MSL type

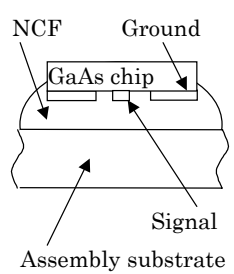

(b) CPW type

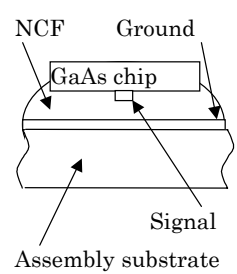

(c) IMSL type
Figure 2: Cross-sectional view of the transmission lines for GaAs chip in TEGs

\subsection{TRANSMISSION LINE TYPE}

Figure 2 shows the cross-sectional view of the transmission lines for GaAs chip in TEGs. We prepared three types of transmission line for the chip in TEGs. The line types are MSL, CPW, and inverted microstrip line (IMSL). In the case of the MSL-type TEG, the signal line is located on the obverse side of the chip and the ground metal pattern is located on the reverse side of the chip, and so the ground current pass is long. In the case of the CPW-type TEG, both the signal line and the ground metal pattern are located on the obverse side of the chip, and so the ground current pass is shorter than that for the MSL type. In the case of the IMSL-type TEG, the signal line is located on the obverse side of the chip and the ground metal pattern is located on the obverse side of the substrate, and so the ground current pass is the shortest among the three types.

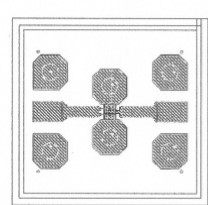

(a) MSL type

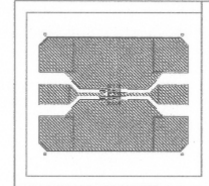

(b) $C P W$ type

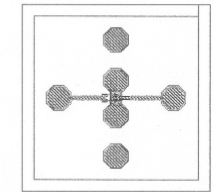

(c) IMSL type
Figure 3: GaAs chip layouts for TEGs

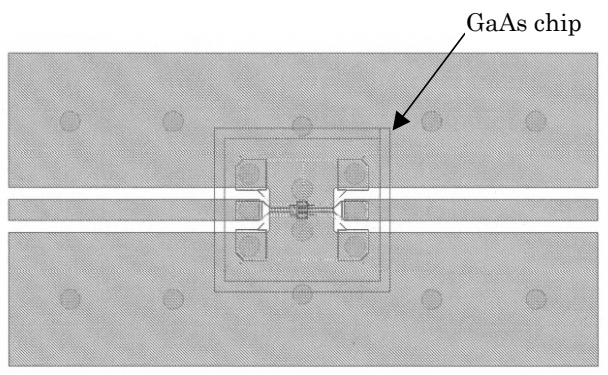

Figure 4: Top view of assembly substrate layout for TEG

\subsection{HEMT TEG STRUCTURE}

The probes contact the surface of the assembly substrate for TEG. All TEGs have extension lines of the same length on the substrates, and the length is $1.0 \mathrm{~mm}$. There is a metal pattern for the ground under the HEMT in the chip and the obverse of the substrate. The bumps were made of gold. A non-conductive-film (NCF) is utilized as underfill resin. Figure 3 shows the GaAs chip layouts for TEGs. The chip sizes are $0.85 \mathrm{~mm} \times 0.8 \mathrm{~mm}$ for the MSL and CPW types, and $0.8 \mathrm{~mm} \times 0.8 \mathrm{~mm}$ for the IMSL type. The chip thickness is $70 \mathrm{um}$. The HEMTs were fabricated with a gate length of $0.25 \mathrm{um}$ and a gate width of $100 \mathrm{um}$. The source electrode of HEMT is connected with the ground. Figure 4 shows the top view of assembly substrate layout for TEG. The substrate material is an alumina ceramic, and its thickness is $200 \mathrm{um}$. A CPW with reverse side ground is 
utilized as the transmission line for the assembly substrate.

\section{CHARACTERIZATION}

The characteristics of the HEMT TEGs using flip-chip interconnections were evaluated. We measured the two-port scattering parameters from $0.5 \mathrm{GHz}$ to 40.0 GHz. The reference plane was set at the edge of the coplanar waveguide on the assembly substrate, which is the probe contact position, using short-open-load-through (SOLT) calibration. The HEMT TEG operated with a drain voltage of $6.0 \mathrm{~V}$ and a drain current of $15 \mathrm{~mA}$.

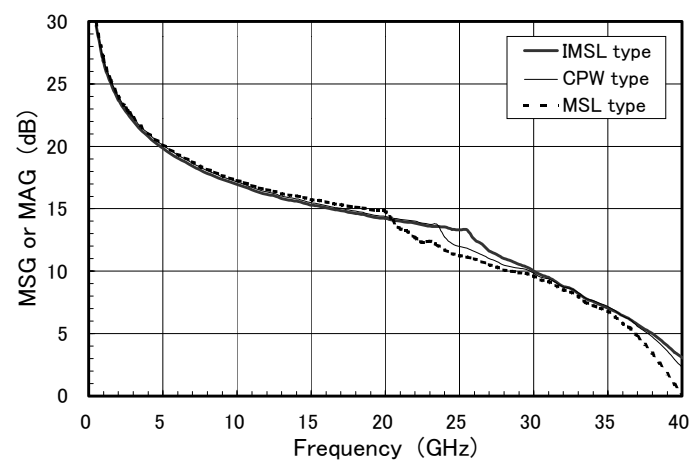

(a) $M S G$ or $M A G$

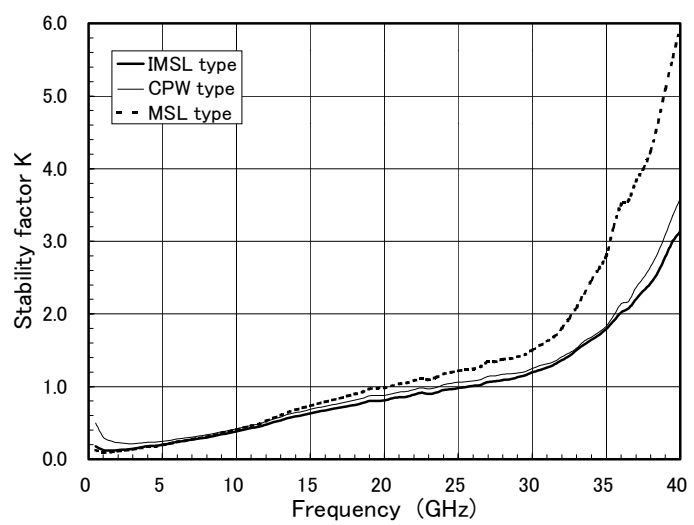

(b) Stability factor $K$

Figure 5: Measured transmission characteristics of the HEMT TEGs with flip-chip interconnections

Figure 5 shows the measured transmission characteristics of the HEMT TEGs with flip-chip interconnections, Figure 6 shows the measured reflection characteristics of the HEMT TEGs with flip-chip interconnections, and Table 1 shows the characteristics of the HEMT TEGs at $30 \mathrm{GHz}$. All three types of TEGs with different transmission line types achieved high performance and had similar values of a maximum available power gain (MAG) at $30 \mathrm{GHz}$. However, in the high-frequency ranges which approach $40 \mathrm{GHz}$, the values of an MAG for the IMSL-type TEG are the highest, and those for the MSL-type TEG are the lowest among the three types of TEGs. We suppose that the long ground current pass causes the inductor between the source electrode of HEMT and the real ground for TEG to increase, and so the characteristics of TEG with long ground current pass are inferior. The MAG values of the HEMT at $40 \mathrm{GHz}$ were $3.11 \mathrm{~dB}$ for the IMSL-type TEG, $2.31 \mathrm{~dB}$ for the CPW-type TEG, and $0.12 \mathrm{~dB}$ for the MSL-type TEG.

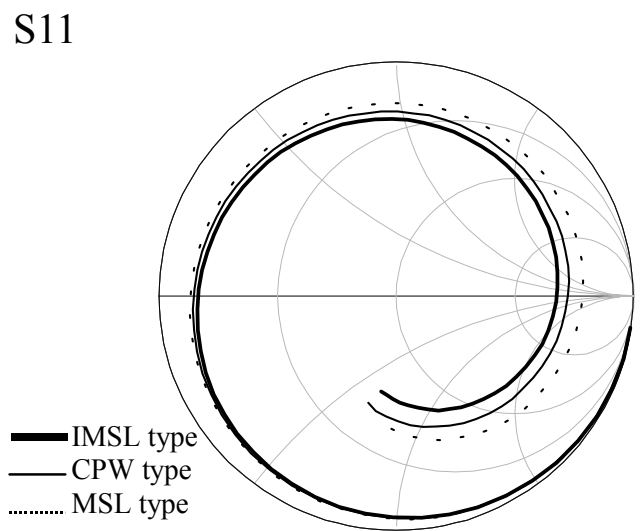

Frequency $0.5 \mathrm{GHz}$ to $40 \mathrm{GHz}$

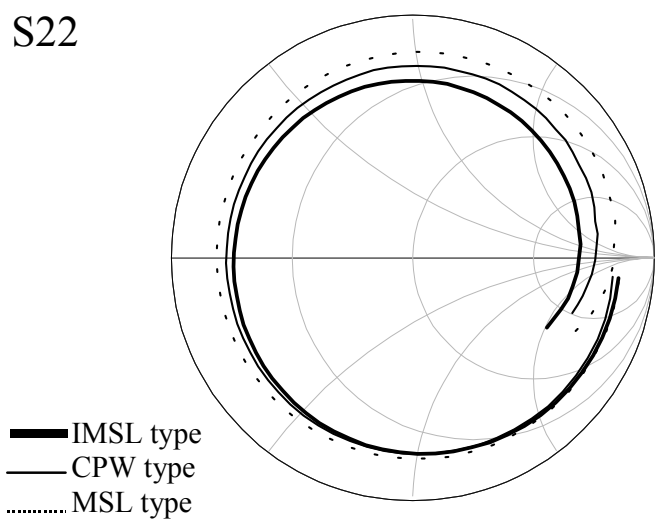

Frequency $0.5 \mathrm{GHz}$ to $40 \mathrm{GHz}$

Figure 6: Measured reflection characteristics of the HEMT TEGs with flip-chip interconnections

\section{CONCLUSION}

We have developed a GaAs HEMT with flip-chip interconnections. There are various ground current passes for the HEMT on surface of the GaAs chip in this assembly structure, each pass depending on the transmission line type for the chip. We evaluated the high-frequency characteristics of the HEMT TEGs with 
flip-chip interconnection for three types, that is, with an MSL, with a CPW, and with an IMSL for the chip. All three types of TEGs had similar values of an MAG at 30 GHz. However, the IMSL-type TEG, which had superior characteristics in high-frequency ranges of more than $30 \mathrm{GHz}$, was chosen as the most suitable type. The IMSL-type TEG had an MAG of $10.02 \mathrm{~dB}$ and a Rollett stability factor $\mathrm{K}$ of 1.20 at $30 \mathrm{GHz}$.

Table 1

\section{Characteristics of the HEMT TEGs}

(@Freq.=30GHz)

\begin{tabular}{|c|c|c|c|}
\hline & MSL type & CPW type & IMSL type \\
\hline MAG (dB) & 9.60 & 9.83 & 10.02 \\
\hline $\mathrm{K}$ & 1.50 & 1.25 & 1.20 \\
\hline $\begin{array}{l}\text { S11 Mag. } \\
\text { Ang. (deg) }\end{array}$ & $\begin{array}{l}0.79 \\
1.92\end{array}$ & $\begin{array}{c}0.72 \\
-0.09\end{array}$ & $\begin{array}{l}0.67 \\
-2.19\end{array}$ \\
\hline $\begin{array}{l}\text { S22 Mag. } \\
\text { Ang. (deg) }\end{array}$ & $\begin{array}{c}0.86 \\
52.37\end{array}$ & $\begin{array}{c}0.8 \\
63.26\end{array}$ & $\begin{array}{c}0.73 \\
65.04\end{array}$ \\
\hline
\end{tabular}

\section{ACKNOWLEDGEMENTS}

The authors wish to thank Y. Fuchida and A. Ishimaru for various technical contributions. They are also grateful to S. Kamihashi, Dr. H. Yoshinaga and Dr. $H$. Tsurumi for their encouragement throughout this work.

\section{REFERENCES}

(1) T. Hirose, K. Makiyama, et al., "A flip-chip MMIC design with coplanar waveguide transmission line in the W-band," IEEE Trans. Microwave Theory \& Tech. vol. 46, No.12, pp. 2276-2282, Dec. 1998.

(2) K. Maruhashi, M. Ito, et al., "Low-cost 60GHzband antenna-integrated transmitter/receiver modules utilizing multi-layer low-temperature co-fired ceramic technology," IEEE ISSCC Digest of Technical Papers, pp. 324-325, 2000

(3) K. Takahashi, S. Fujita, et al., "K-band receiver front-end IC integrating micromachined filter and flipchip assembled active devices," IEEE Microwave Theory \& Tech. Symp. Digest, pp. 229-232, 1999.

(4) T. Shimura, Y. Ohashi, "A Single-chip transceiver module for 76-GHz automotive radar sensors," 31th EuMC Digest, vol.3, pp.153-156, 2001.

(5)

O. Vendier, J-P. Fraysse, et al., "Flip-chip mounted, Ku band power amplifier compliant with space applications," IEEE Microwave Theory \& Tech. Symp. Digest, pp. 1389-1392, 2002.
(6)

K. Suzuki, H. Sato, et al., "Compact ODU with dielectric diplexer for FWA (fixed wireless access)," TSMMW Tech. Digest, pp.129-132, 2000.

(7) N. Ono, H. Hirai, et al., "GaAs HEMT MMICs with flip-chip interconnections using underfill resin," ISMOT Proc., pp.285-288, 2001. 